\title{
Potential impacts of a novel integrated extracorporeal-CPR workflow using an interventional radiology and immediate whole-body computed tomography system in the emergency department
}

\author{
Kei Hayashida ${ }^{1 *}\left(\mathbb{D}\right.$, Takahiro Kinoshita ${ }^{2}$, Kazuma Yamakawa ${ }^{2}$, Santiago J. Miyara ${ }^{1,3}$, Lance B. Becker ${ }^{1}$ and
} Satoshi Fujimi ${ }^{2}$

\begin{abstract}
Extracorporeal cardiopulmonary resuscitation (ECPR) can be associated with increased survival and neurologic benefits in selected patients with out-of-hospital cardiac arrest (OHCA). However, there remains insufficient evidence to recommend the routine use of ECPR for patients with OHCA. A novel integrated trauma workflow concept that utilizes a sliding computed tomography (CT) scanner and interventional radiology (IR) system, named a hybrid emergency room system (HERS), allowing emergency therapeutic interventions and CT examination without relocating trauma patients, has recently evolved in Japan. HERS can drastically shorten the ECPR implementation time and more quickly facilitate definitive interventions than the conventional advanced cardiovascular life support workflow. Herein, we discuss our novel workflow concept using HERS on ECPR for patients with OHCA.
\end{abstract}

Keywords: Out-of-hospital cardiac arrest, Extracorporeal cardiopulmonary resuscitation, Emergency department, HERS, Concurrent treatment

Extracorporeal cardiopulmonary resuscitation (ECPR) can restore blood circulation in patients with out-ofhospital cardiac arrest (OHCA), as the cardiopulmonary circuit allows immediate stabilization via effective perfusion and gas exchange. Recently, ECPR devices are becoming smaller and cheaper, providing an opportunity to install this rescue therapy in the emergency department (ED) [1]. The international resuscitation guideline mentioned ECPR as a potential support to be considered in settings where it can be rapidly implemented, and for selected patients with a suspected etiology of cardiac arrest that is potentially reversible during a limited period of mechanical cardiorespiratory support [2]. However,

\footnotetext{
* Correspondence: keilinda0714@gmail.com

${ }^{1}$ Department of Emergency Medicine, Feinstein Institutes for Medical Research, Northwell Health System, 350 Community Dr, Manhasset, NY 11030, USA

Full list of author information is available at the end of the article
}

there remains insufficient evidence to recommend the routine use of ECPR for patients with OHCA [2].

In 2011, a new integrated trauma workflow concept, with a sliding computed tomography scanner (CT) and interventional radiology (IR) system that enables CT examination and emergency therapeutic intervention, was implemented in Japan. It contains a carbon-fiber fluoroscopic table and all the necessary equipment for life-saving procedures, including airway management, CT examinations, angioembolization, and emergency surgery, which can be performed on the same table (Fig. 1). This integral structure allows physicians to perform both examinations and rapid safe effective procedures, without relocating the patient and is called a hybrid emergency room system (HERS) [3, 4]. We previously demonstrated that the HERS is associated with a decreased mortality in patients with severe blunt trauma compared to that with conventional trauma management, after accounting for potential confounders (adjusted odds

(c) The Author(s). 2020 Open Access This article is distributed under the terms of the Creative Commons Attribution 4.0 International License (http://creativecommons.org/licenses/by/4.0/), which permits unrestricted use, distribution, and 


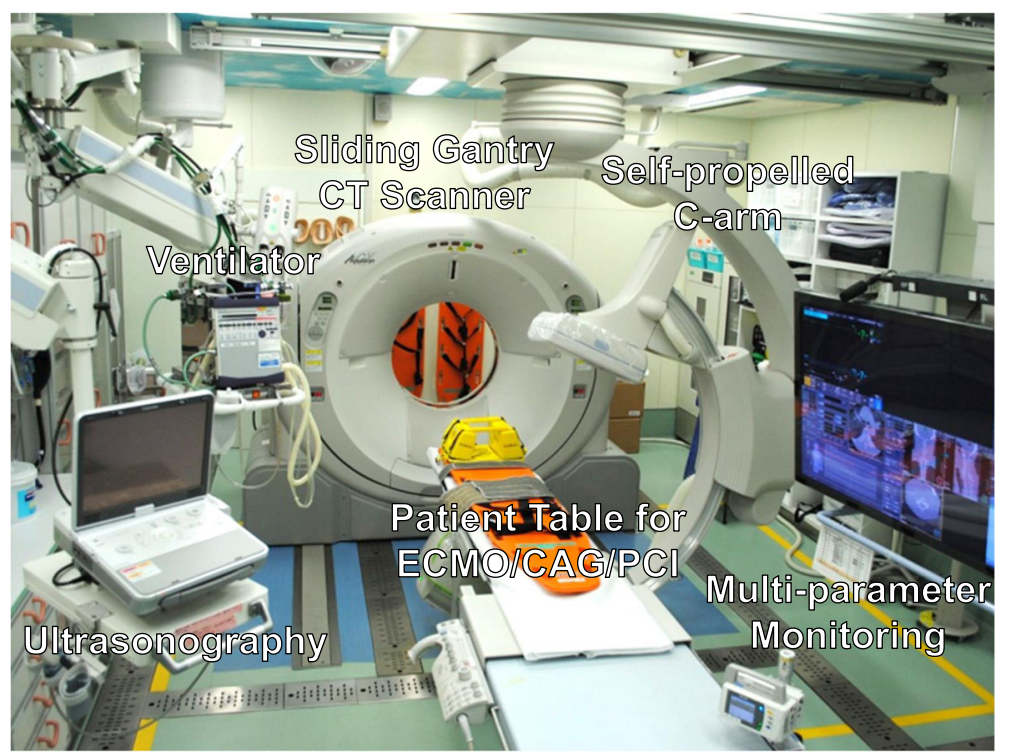

Fig. 1 A photograph showing our IR-CT system in the emergency department. All life-saving procedures including airway management, emergency surgery, and transcatheter arterial embolization can be performed on the table without relocating the patient

ratio [OR], 0.50; 95\% confidence interval [CI], 0.29-0.85; $p=0.011$ [ [4]. Patients treated with the HERS had shorter time intervals from ER arrival to CT examination and emergency surgery compared to those for conventional management (median [25th-75th percentiles]: 11 [8-16] $\min$ vs. 26 [21-32] min, $p<0.0001 ; 47$ [37-57] min vs. 68 [51-85] min, $\mathrm{p}<0.0001$, respectively) [4]. These beneficial observations contributed to the rapid spread of the HERS concept; as of September 2019, eleven tertiary emergency hospitals in Japan and one trauma center in Korea have installed a HERS. This system could be well adapted to ECPR, providing an expedient initiation of mechanical cardiopulmonary support for patients with non-traumatic OHCA.

Immediate high-quality $\mathrm{CPR}$, providing effective oxygenation to the vital organs until ROSC is achieved, is crucial for optimal outcomes. However, there is no consensus regarding the CPR time interval before ECPR implementation. In a single-center retrospective cohort study, the impact of CPR duration on neurological outcomes was evaluated in patients with OHCA; ROSC occurred within 16 min of CPR in $89.7 \%$ of patients with good neurological outcomes [5]. In their study, the probability of good functional recovery among all CPR attempts fell to less than $2 \%$ when the CPR duration was over $15 \mathrm{~min}$. In another retrospective study including 3992 OHCA patients with resuscitation attempts, refractory OHCA, age, public $\mathrm{OHCA}$, witnessed OHCA, bystander CPR performed, high severity of comorbidity, and resuscitation length $>30 \mathrm{~min}$ were all found to be independently associated with increased 30-day mortality. Comparing all patients with a duration of resuscitation $>30$ min with duration $<30 \mathrm{~min}$, they found 30-day survival of 38 and $50 \%$, respectively $(P<0.001)$ [6]. In addition, a longer pre-ECPR resuscitation time is significantly associated with a higher mortality rate in patients with OHCA $[7,8]$. These currently available data do not suggest that a longer pre-ECPR resuscitation time is a contraindication to ECPR; rather, ECPR should be immediately available to patients who have failed to respond to conventional CPR within the first 30 min. Moreover, as it is reported that the median prehospital resuscitation duration is approximately $30 \mathrm{~min}$ [9], ECPR flow should commence for selected patients immediately after hospital arrival, if the patient fails to respond to a short interval of conventional advanced cardiovascular life support (ACLS) in the ED.

Yannopoulos and colleagues [10] evaluated a novel protocol with early transport to a cardiac catheterization laboratory (CCL) for ECPR and revascularization in OHCA patients with refractory ventricular fibrillation/ ventricular tachycardia. In patients who received the early transport protocol, the average time from the 911 call to CCL arrival was $58 \pm 17 \mathrm{~min}, 28 / 62(45 \%)$ patients survived to hospital discharge, and 26 (42\%) had functionally favorable outcomes. In contrast, only 26/170 (15.3\%) patients in a historical routine care group survived to hospital discharge with a favorable outcome [OR 4.0, 95\%CI 2.08-7.7] [10]. These observations suggest that, in these patients, the early initiation of ECPR to allow for coronary angiography (CAG) and percutaneous coronary intervention (PCI) would likely be more effective than continuing non-invasive conventional ACLS alone.

For treatable etiologies, the objective of ECPR is primarily to increase the chance of ROSC and improve the 
hemodynamic status, leading to the concept of "treatment bundles" to preserve intact neurological function and improve the long-term prognosis. The treatments for the cause of cardiac arrest should be planned under a timeconscious algorithm. Patients with ROSC and suspected ST-segment elevation myocardial infarction should be considered to receive CAG and possible immediate PCI. If a massive/submassive pulmonary embolism (PE) is likely the etiology, CT pulmonary angiography should be considered. Although the optimal timing of treatment for acute PE remains unclear, due to the absence of randomized controlled trials, subsequent definitive therapies (i.e. thrombolytic therapy, or surgical/catheter embolectomy if thrombolytic therapy is contraindicated or failed) should be performed as soon as possible. If an acute ischemic stroke/intracranial hemorrhage is suspected as a precipitant, head CT should be performed for further interventions. Immediate brain CT after ROSC in patients treated with therapeutic hypothermia can help predict the outcome $[11,12]$.

The schematic concept of HERS integrated workflow is described in Fig. 2. The HERS enables ACLS to be followed seamlessly by ECPR induction and post-ECPR procedures, including CAG and $\mathrm{PCI}$, without any patient transfer. The HERS likely shortens the time from the 911 call to ECPR initiation and improve survival in OHCA, as early transport contributes to improved outcomes. In addition, the HERS enables safe head and body CT examinations during the post-ECPR phase, even in hemodynamically unstable patients, while inhospital transport with an extracorporeal membrane oxygenation (ECMO) device implantation can cause considerable harm. For example, when the coronary artery is recognized as intact after ECPR induction followed by CAG, the resuscitation team can seamlessly perform whole-body CT to reveal other treatable etiologies. Vascular complications of ECMO constitute the most important aspect as far as treatment outcomes are concerned [13]. Fluoroscopy with the C-arm allows for immediate and safe cannulations into the femoral artery and vein during ongoing CPR, therefore the HERS might diminish the rates of vascular complications from ECMO placement, including failure to cannulate, accidental cannula displacement, vessel injury, limb ischemia, and retroperitoneal bleeding. Thus, given that an extremely time-conscious algorithm should be adopted in the management of, not only severe trauma but also life-threatening critical illnesses mentioned above, the HERS can provide benefits for cardio-cerebral resuscitation in patients with OHCA or severe medical conditions.

Since no/low-flow time intervals are crucial determinants of the outcome, all efforts should be made to minimize the time from cardiac arrest to ECMO flow initiation. Our novel workflow concept using the HERS can drastically shorten the ECPR implementation delay and more quickly facilitate definitive interventions than the conventional ACLS workflow, without relocating the patients. ECPR with the HERS may exponentially accelerate improvements in cardiac arrest management. Further randomized controlled trials with strict inclusion criteria and an appropriate treatment protocol will be required to demonstrate the impact of HERS on ECPR for patients with OHCA.

\section{Conventional E-CPR Workflow}

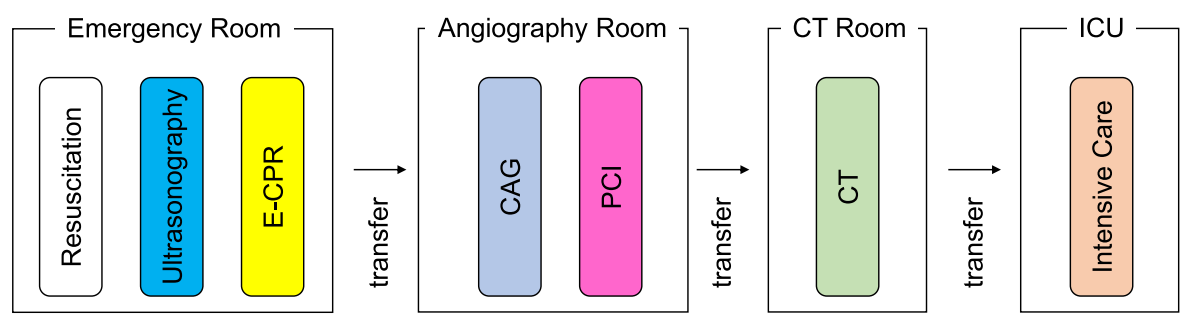

Novel E-CPR Workflow Using the Hybrid ER
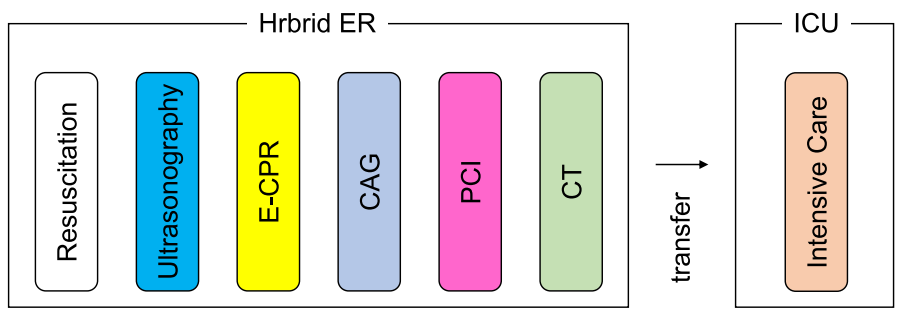

Fig. 2 The schematic integrated concept of a hybrid emergency room system (HERS) workflow 


\section{Abbreviations}

ACLS: Advanced cardiovascular life support; CAG: Coronary angiography;

CCL: Catheterization laboratory; Cl: Confidence interval;

CPR: Cardiopulmonary resuscitation; CT: Computed tomography;

ECMO: Extracorporeal membrane oxygenation; ECPR: Extracorporeal cardiopulmonary resuscitation; ED: Emergency department; HERS: Hybrid emergency room system; IR: Interventional radiology; OHCA: Out-of-hospital cardiac arrest; OR: Odds ratio; PCl: Percutaneous coronary intervention;

PE: Pulmonary embolism; ROSC: Return of spontaneous circulation

\section{Acknowledgments}

The authors wish to acknowledge the members of the Japanese Association for Hybrid Emergency Room System (JA-HERS) Scientific Promotion Committee.

\section{Authors' contributions}

Drafting manuscript: $\mathrm{KH}$. Critical version of the manuscript for important intellectual content: TK, KY, SJM, LBB and SF. All authors have read and approved the manuscript.

\section{Funding}

No funding was obtained for this study.

\section{Availability of data and materials}

The data described in the current manuscript are available from the

corresponding author on reasonable request.

\section{Ethics approval and consent to participate}

Not applicable.

\section{Consent for publication}

Not applicable.

\section{Competing interests}

The authors declare that they have no competing interests.

\section{Author details}

'Department of Emergency Medicine, Feinstein Institutes for Medical Research, Northwell Health System, 350 Community Dr, Manhasset, NY 11030, USA. ${ }^{2}$ Division of Trauma and Surgical Critical Care, Osaka General Medical Center, 3-1-56 Bandai-Higashi, Sumiyoshi-ku, Osaka 558-8558, Japan. ${ }^{3}$ Elmezzi Graduate School of Molecular Medicine, Manhasset, NY, USA.

Received: 21 November 2019 Accepted: 8 January 2020

Published online: 16 January 2020

\section{References}

1. Tonna JE, Selzman CH, Mallin MP, et al. Development and implementation of a comprehensive, multidisciplinary emergency department extracorporeal membrane oxygenation program. Ann Emerg Med. 2017;70: 32-40.

2. Link MS, Berkow LC, Kudenchuk PJ, et al. Part 7: adult advanced cardiovascular life support: 2015 American Heart Association guidelines update for cardiopulmonary resuscitation and emergency cardiovascular care. Circulation. 2015;132:S444-64.

3. Wada D, Nakamori Y, Yamakawa K, Fujimi S. First clinical experience with IVR-CT system in the emergency room: positive impact on trauma workflow. Scand J Trauma Resusc Emerg Med. 2012;20:52.

4. Kinoshita T, Yamakawa K, Matsuda H, Yoshikawa Y, Wada D, Hamasaki T, Ono K, Nakamori Y, Fujimi S. The survival benefit of a novel trauma workflow that includes immediate whole-body computed tomography, surgery, and interventional radiology, all in one trauma resuscitation room: a retrospective historical control study. Ann Surg. 2019;269:370.

5. Reynolds JC, Frisch A, Rittenberger JC, Callaway CW. Duration of resuscitation efforts and functional outcome after out-of-hospital cardiac arrest: when should we change to novel therapies? Circulation. 2013;128 2488-94.

6. Gregers E, Kjærgaard J, Lippert F, Thomsen JH, Køber L, Wanscher M, Hassager C, Søholm H. Refractory out-of-hospital cardiac arrest with ongoing cardiopulmonary resuscitation at hospital arrival - survival and neurological outcome without extracorporeal cardiopulmonary resuscitation. Crit Care. 2018:22:242

7. Leick J, Liebetrau C, Szardien S, Fischer-Rasokat U, Willmer M, van Linden A, Blumenstein J, Nef H, Rolf A, Arlt M, Walther T, Hamm C, Mollmann H. Doorto-implantation time of extracorporeal life support systems predicts mortality in patients with out-of-hospital cardiac arrest. Clin Res Cardiol. 2013;102:661-9.

8. Johnson NJ, Acker M, Hsu CH, Desai N, Vallabhajosyula P, Lazar S, Horak J, Wald J, McCarthy F, Rame E, Gray K, Perman SM, Becker L, Cowie D, Grossestreuer A, Smith T, Gaieski DF. Extracorporeal life support as rescue strategy for out-of-hospital and emergency department cardiac arrest. Resuscitation. 2014;85:1527-32

9. Nagao K, Nonogi H, Yonemoto N, Gaieski DF, Ito N, Takayama M, Shirai S, Furuya S, Tani S, Kimura T. Saku K; Japanese Circulation Society with resuscitation science study (JCS-ReSS) group. Duration of Prehospital resuscitation efforts after out-of-hospital cardiac arrest. Circulation. 2016;133: 1386-96.

10. Yannopoulos D, Bartos JA, Raveendran G, Conterato M, Frascone RJ, Trembley A, John R, Connett J, Benditt DG, Lurie KG, Wilson RF, Aufderheide TP. Coronary artery disease in patients with out-of-hospital refractory ventricular fibrillation cardiac arrest. J Am Coll Cardiol. 2017;70:1109-17.

11. Kim SH, Choi SP, Park KN, Youn CS, Oh SH, Choi SM. Early brain computed tomography findings are associated with outcome in patients treated with therapeutic hypothermia after out-of-hospital cardiac arrest. Scand J Trauma Resusc Emerg Med. 2013;21:57.

12. Langkiær S, Hassager C, Kjaergaard J, Salam I, Thomsen JH, Lippert FK, Wanscher M, Køber L, Nielsen N, Søholm H. Prognostic value of reduced discrimination and oedema on cerebral computed tomography in a daily clinical cohort of out-of-hospital cardiac arrest patients. Resuscitation. 2015; 92:141-7.

13. Pillai AK, Bhatti Z, Bosserman AJ, Mathew MC, Vaidehi K, Kalva SP. Management of vascular complications of extra-corporeal membrane oxygenation. Cardiovasc Diagn Ther. 2018;8:372-7.

\section{Publisher's Note}

Springer Nature remains neutral with regard to jurisdictional claims in published maps and institutional affiliations.
Ready to submit your research? Choose BMC and benefit from:

- fast, convenient online submission

- thorough peer review by experienced researchers in your field

- rapid publication on acceptance

- support for research data, including large and complex data types

- gold Open Access which fosters wider collaboration and increased citations

- maximum visibility for your research: over $100 \mathrm{M}$ website views per year

At BMC, research is always in progress.

Learn more biomedcentral.com/submission 